To appear in Integral Transforms and Special Functions

Vol. 00, No. 00, , 1-11

\title{
Monotonicity of zeros of polynomials orthogonal with respect to an even weight function
}

\author{
K. Jordaan ${ }^{a}$, H. Wang ${ }^{b *}$ and J. Zhou ${ }^{c}$ \\ ${ }^{a}$ Department of Mathematics and Applied Mathematics, University of Pretoria, Lynnwood \\ Road, Pretoria, 0002, South Africa; ${ }^{b}$ School of Mathematics and Physics, University of South \\ China, Hengyang 421001, Hunan, P. R. China ; Department of Mathematics, Foshan \\ University, Foshan 528000, Guangdong, P. R. China
}

(5 September 2013)

\begin{abstract}
The monotonicity properties of all the zeros with respect to a parameter of orthogonal polynomials associated with an even weight function are studied. The results we obtain extend the work of A. Markoff. The monotonicity of the zeros of Gegenbauer, Freud-type and symmetric Meixner-Pollaczek orthogonal polynomials as well as Al-Salam-Chihara $q$-orthogonal polynomials are investigated. For the Meixner-Pollaczek polynomials, a special case of a conjecture by Jordaan and Toókos which concerns the interlacing of their zeros between two different sequences of Meixner-Pollaczek polynomials is proved.
\end{abstract}

Keywords: Freud-type orthogonal polynomial; Meixner-Pollaczek polynomials;

Gegenbauer polynomials; Al-Salam-Chihara polynomials; zeros; monotonicity; interlacing

AMS Subject Classification: 33C45; 33C52; 42C05

\section{Introduction}

In 1886, an important result about the monotonicity properties of zeros of orthogonal polynomials with respect to a parameter was obtained by A. Markoff (cf. [1], [14, Thm. 6.12.1]). Since then, related problems have been studied extensively.

The monotonicity of all the zeros as well as the extreme zeros of polynomials satisfying recursion formulas, referred to as birth and death processes, were considered in [2-4] using a finite dimensional version of the Hellman-Feynman Theorem. Refer to [5] for a summary of these results and [6] together with the list of references in [6] for more applications. The result obtained in [2, Thm. 4] by making use of the Hellman-Feynman Theorem can be stated as follows.

Lemma 1.1. [5, Thm. 7.3.6] If $\left\{p_{n}\right\}_{n=0}^{\infty}$ is a sequence of polynomials satisfying the recurrence relation

$$
x a_{n}(\tau) p_{n}(x)=p_{n+1}(x)+p_{n-1}(x),
$$

with $p_{0}(x)=1, p_{1}(x)=x$, then the positive zeros of $p_{n}(x)$ are decreasing (increasing) function of $\tau$ when $a_{n}(\tau)$ is an increasing (decreasing) function of $\tau$.

\footnotetext{
${ }^{*}$ Corresponding author. Email: sysuwhylj@aliyun.com
} 
The problem of finding the extremal function $f(s)$ that forces the product $f(s) x_{n, k}^{s}$, $k=1,2, \ldots, n$ to reverse the monotonicity with respect to the parameter $s$ for the ze$\operatorname{ros} x_{n, k}^{s}$ of an $n$-th degree polynomial dependant on parameter $s$, was investigated in [7-13] using various techniques involving, amongst others, Sturm Liouville differential equations, Sturm's theorem (cf. [14, Thm. 1.82.1] and the Routh-Hurwitz criterion. The monotonicity of all the zeros of polynomials satisfying second order ordinary linear differential equations is discussed in $[15,16]$.

The manner in which the zeros of a polynomial change as the parameter changes can be used to study comparison and interlacing properties of the zeros [17-21]. Markoff's theorem can be used to show that the zeros of classical orthogonal polynomials like Laguerre and Jacobi polynomials are monotone functions of the parameter(s) involved by using the derivative of the weight function with respect to the parameter(s). A slightly generalised version of Markoff's theorem, stated as an exercise in [22, Chap. 3, ex. 15] and proved in [5, Thm. 7.1.1] (see also [23, Thm. 1]) can be applied to discrete orthogonal polynomials such as Meixner and Hahn polynomials as well as orthogonal Laurent polynomials. However, weight functions of orthogonal polynomials are not always easy to compute and even when they are known, they do not necessarily satisfy the conditions of Markoff's theorem and its generalisations, especially if the weight function changes monotonicity on the interval of orthogonality or, as in the examples in [24-26], the measure of orthogonality has discrete parts where the location of the masses depends on the parameter.

In this paper, we extend the result of Markoff, to cases where the weight function is even. It is well-known in this case that the zeros of the corresponding orthogonal polynomials are symmetric about the origin which implies that the positive and the negative zeros have opposing monotonicity and the result of Markoff and its generalisation no longer apply. As a result of this symmetry, it suffices to study only the monotonicity and interlacing of the positive zeros. A classical example is Gegenbauer polynomials which have the weight function $w(x ; \alpha)=\left(1-x^{2}\right)^{\alpha-1 / 2}, \alpha>1, x \in(-1,1)$ that changes monotonicity at the origin. If $\left\{x_{\nu}=x_{\nu}(\alpha)\right\}$ denote the zeros of the Ultraspherical polynomial in decreasing order, then

$$
\frac{\partial x_{\nu}}{\partial \alpha}<0, \quad \nu=1,2, \ldots,[n / 2] .
$$

The proof of Szegö [14, Thm. 6.21.1] was based on the relation between Ultraspherical polynomials and Jacobi polynomials (cf. [14, (6.21.2)]) whereas Stieltjes proved it from the differential equation (cf. [27, p. 77]).

The structure of our paper is as follows. In Section 2 we state and prove the main results. Section 3 provides various applications of the results. These include the monotonicity properties of zeros of, for example, Freud-type orthogonal polynomials and Al-SalamChihara polynomials. Furthermore, interlacing properties of zeros of symmetric MeixnerPollaczek polynomials are considered which yield a proof of a special case of a conjecture pointed out by Jordaan and Toókos in [28].

\section{Main results}

Theorem 2.1 is analogous to [5, Thm. 7.1.1] and utilises some specific properties of orthogonal polynomials whose weight function is even.

Theorem 2.1. Let $\left\{p_{n}(x, \tau)\right\}_{n=0}^{\infty}$ be orthogonal with respect to $d \alpha(x, \tau)=w(x, \tau) d \alpha(x)$ where $w(x, \tau)$ is an even function on the interval $[-a, a], a>0$ depending on a parameter 
$\tau$ such that $w(x, \tau)$ is positive and continuous for $-a<x<a, \tau_{1}<\tau<\tau_{2}$. Also, suppose that the partial derivative $w_{\tau}(x, \tau)$ for $-a<x<a, \tau_{1}<\tau<\tau_{2}$ exists and is continuous, and the integrals

$$
\int_{-a}^{a} x^{\nu} w_{\tau}(x, \tau) d \alpha(x), \nu=0,1,2, \ldots, 2 n-1
$$

converge uniformly in every closed interval $\left[\tau^{\prime}, \tau^{\prime \prime}\right] \subset\left(\tau_{1}, \tau_{2}\right)$. If the positive zeros of $p_{n}(x, \tau)$ are denoted by $x_{1}(\tau)>x_{2}(\tau)>\cdots>x_{[n / 2]}(\tau)>0$, where $[x]$ denotes the largest integer smaller than $x$, then the $\nu$ th zero $x_{\nu}(\tau)$ (for a fixed value of $\nu$ ) is an increasing (decreasing) function of $\tau$ provided that $w_{\tau} / w$ is an increasing (decreasing) function of $x, 0 \leq x<a$.

Proof. The mechanical quadrature formula (cf. [5, (2.4.1)])

$$
\int_{-a}^{a} \rho(x) d \alpha(x, \tau)=\sum_{\nu=1}^{n} \lambda_{\nu}(\tau) \rho\left(x_{\nu}(\tau)\right)
$$

holds for polynomials $\rho(x)$ of degree at most $2 n-1$. Differentiating (3) with respect to $\tau$, we obtain

$$
\int_{-a}^{a} \rho(x) w_{\tau}(x, \tau) d \alpha(x)=\sum_{\nu=1}^{n} \lambda_{\nu}(\tau) \rho^{\prime}\left(x_{\nu}\right) x_{\nu}^{\prime}(\tau)+\sum_{\nu=1}^{n} \lambda_{\nu}^{\prime}(\tau) \rho\left(x_{\nu}\right) .
$$

Now we choose

$$
\rho(x)=\frac{\left\{p_{n}(x, \tau)\right\}^{2}}{x-x_{\nu}}
$$

then, since $x_{\nu}$ is a removable singularity, $\rho^{\prime}\left(x_{\nu}\right)=\left\{p_{n}^{\prime}\left(x_{\nu}, \tau\right)\right\}^{2}$ while $\rho^{\prime}\left(x_{\mu}\right)=0$ if $\mu \neq \nu$ and hence

$$
\int_{-a}^{a} w_{\tau}(x, \tau) \frac{\left\{p_{n}(x, \tau)\right\}^{2}}{x-x_{\nu}} d \alpha(x)=\lambda_{\nu}(\tau)\left\{p_{n}^{\prime}\left(x_{\nu}, \tau\right)\right\}^{2} x_{\nu}^{\prime}(\tau)
$$

In view of the orthogonality the integral

$$
\int_{-a}^{a} \frac{\left\{p_{n}(x, \tau)\right\}^{2}}{x-x_{\nu}} w(x, \tau) d \alpha(x)=0
$$

so (4) can be rewritten as

$$
\int_{-a}^{a}\left\{w_{\tau}(x, \tau)-\frac{w_{\tau}\left(x_{\nu}, \tau\right)}{w\left(x_{\nu}, \tau\right)} w(x, \tau)\right\} \frac{\left\{p_{n}(x, \tau)\right\}^{2}}{x-x_{\nu}} d \alpha(x)=\lambda_{\nu}(\tau)\left\{p_{n}^{\prime}\left(x_{\nu}, \tau\right)\right\}^{2} x_{\nu}^{\prime}(\tau)
$$

In addition, since the weight function $w(x, \tau)$ is even, the corresponding orthogonal polynomials satisfy $p_{2 n-1}(-x, \tau)=-p_{2 n-1}(x, \tau)$ while $p_{2 n}(-x, \tau)=p_{2 n}(-x, \tau)$ for $n=$ $1,2, \ldots$ and therefore $\left\{p_{n}(-x, \tau)\right\}^{2}=\left\{p_{n}(x, \tau)\right\}^{2}$. This, together with the fact that 
$w_{\tau}(x, \tau)$ is also an even functions of $x$, yields

$$
\begin{aligned}
& \int_{-a}^{0}\left\{w_{\tau}(x, \tau)-\frac{w_{\tau}\left(x_{\nu}, \tau\right)}{w\left(x_{\nu}, \tau\right)} w(x, \tau)\right\} \frac{\left\{p_{n}(x, \tau)\right\}^{2}}{x-x_{\nu}} d \alpha(x) \\
& =-\int_{0}^{a}\left\{w_{\tau}(x, \tau)-\frac{w_{\tau}\left(x_{\nu}, \tau\right)}{w\left(x_{\nu}, \tau\right)} w(x, \tau)\right\} \frac{\left\{p_{n}(x, \tau)\right\}^{2}}{x+x_{\nu}} d \alpha(x) .
\end{aligned}
$$

Substituting (6) into (5), we obtain

$$
\int_{0}^{a}\left\{\frac{w_{\tau}(x, \tau)}{w(x, \tau)}-\frac{w_{\tau}\left(x_{\nu}, \tau\right)}{w\left(x_{\nu}, \tau\right)}\right\}\left\{p_{n}(x, \tau)\right\}^{2} \frac{2 x_{\nu} w(x, \tau)}{\left(x-x_{\nu}\right)\left(x+x_{\nu}\right)} d \alpha(x)=\lambda_{\nu}(\tau)\left\{p_{n}^{\prime}\left(x_{\nu}, \tau\right)\right\}^{2} x_{\nu}^{\prime}(\tau) .
$$

The integrand in (7) has a constant sign, so the positivity of the so-called Christoffel numbers $\lambda_{\nu}(\tau)[14$, p. 48] establishes the result.

The following result is a straightforward consequence of Theorem 2.1.

Theorem 2.2. Let $w(x)$ and $W(x)$ be two weight functions on $[-a, a]$, both positive, even and continuous for $x \in[-a, a]$. Let $W(x) / w(x)$ be increasing on $[0, a)$. Then, if $x_{\nu}$ and $X_{\nu}, \nu=1,2, \ldots,[n / 2]$ denote the positive zeros in decreasing order of the orthogonal polynomials of degree $n$ associated with $w(x)$ and $W(x)$ respectively, we have

$$
x_{\nu}<X_{\nu}, \quad \nu=1,2, \ldots,[n / 2] .
$$

Proof. The proof follows along the same lines of the proof of Theorem 6.12.2. in [14].

\section{Application of results}

\subsection{Ultraspherical polynomials}

The weight function of Ultraspherical polynomials is $w(x, \alpha)=\left(1-x^{2}\right)^{\alpha}$. Since the ratio $\frac{w_{\alpha}}{w}=\log \left(1-x^{2}\right)$ is decreasing for $x \in[0,1)$, (2) can be obtained directly from Theorem 2.1 .

\section{$3.2 \quad$ Freud-type polynomials}

The advantage of our approach can also be illustrated by considering the family $\left\{p_{n}(x)\right\}$, $n \in \mathbb{N}$, of polynomials which are orthonormal with respect to the Freud-type weight

$$
w(x ; t)=\sqrt{\frac{2}{t}} \frac{1}{K_{1 / 4}\left(t^{2} / 2\right)} \exp \left(-x^{4}+t x^{2}-\frac{t^{2}}{4}\right), x \in(-\infty,+\infty), t>0,
$$

where $K_{\nu}(z)$ denotes the Bessel function of the second kind. The polynomials $p_{n}(x)$ satisfy the recurrence relation (cf. [29])

$$
x p_{n}(x)=a_{n+1} p_{n+1}(x)+a_{n} p_{n-1}(x),
$$

where the coefficients $a_{n}$ are recursively defined by the so-called string equation

$$
n=4 a_{n}^{2}\left(a_{n+1}^{2}+a_{n}^{2}+a_{n-1}^{2}-t\right), n \in \mathbb{N} .
$$


The explicit expression of $a_{n}(\tau)$ in (1) cannot be obtained in an obvious way and therefore it seems difficult to make use of Lemma 1.1 to obtain the monotonicity of the zeros of $p_{n}(x)$. However, using Theorem 2.1 we have the following theorem.

Theorem 3.1. Let $\left\{x_{\nu}=x_{\nu}(t)\right\}$ be the zeros of the Freud-type polynomials, orthonormal with respect to the weight (8), in decreasing order, then

$$
\frac{\partial x_{\nu}}{\partial t}>0, \quad \nu=1,2, \ldots,[n / 2]
$$

Proof. It is easy to check that

$$
\ln w(x ; t)=\frac{1}{2}(\ln 2-\ln t)-\ln \left(K_{1 / 4}\left(t^{2} / 2\right)\right)-x^{4}+t x^{2}-\frac{t^{2}}{4}
$$

Differentiating (9) with respect to $t$, we have

$$
\frac{\partial \ln w(x ; t)}{\partial t}=-\frac{1}{2 t}-\frac{t K_{1 / 4}^{\prime}\left(t^{2} / 2\right)}{K_{1 / 4}\left(t^{2} / 2\right)}+x^{2}-\frac{t}{2},
$$

which is an increasing function of $x$ for $x \in(0,+\infty)$. Hence, we can conclude from Theorem 2.1 that the positive zeros of $p_{n}(x)$ increase as a function of $t$, for $t \in(0,+\infty)$. This completes the proof.

\subsection{Symmetric Meixner-Pollaczek polynomials}

For the sake of brevity, we denote the symmetric Meixner-Pollaczek polynomials in the present paper by $P_{n}^{\lambda}(x)$ instead of the standard notation $P_{n}^{\lambda}(x, \pi / 2) . P_{n}^{\lambda}(x)$ are defined by (cf. [14])

$$
P_{n}^{\lambda}(x)=\frac{(2 \lambda)_{n}}{n !} e^{\frac{n \pi i}{2}}{ }_{2} F_{1}\left(\begin{array}{c}
-n, \lambda+i x \\
2 \lambda
\end{array} \mid 2\right)
$$

and are orthogonal on $\mathbb{R}$ for $\lambda>0$ with respect to the weight function $w(x, \lambda)=|\Gamma(\lambda+i x)|^{2}$. Substituting the expression (cf. [30, p.72])

$$
\begin{aligned}
& (c+n){ }_{2} F_{1}\left(\begin{array}{c}
-n-1, b+1 \\
c
\end{array} \mid z\right) \\
& =(n+b+1)(1-z){ }_{2} F_{1}\left(\begin{array}{c}
-n, b+1 \\
c
\end{array} \mid z\right)+(c-1-b){ }_{2} F_{1}\left(\begin{array}{c}
-n, b \\
c
\end{array} \mid z\right)
\end{aligned}
$$

into (cf. [31, eqn. (7)])

$$
\begin{aligned}
& \left(\frac{c}{b+n+1}-z\right){ }_{2} F_{1}\left(\begin{array}{c}
-n, b \\
c
\end{array} \mid z\right) \\
& =\frac{c}{(b+n+1)}{ }_{2} F_{1}\left(\begin{array}{c}
-n-1, b+1 \\
c
\end{array} \mid z\right)+\frac{(b-c) n z^{2}}{c(c+1)}{ }_{2} F_{1}\left(\begin{array}{c}
-n+1, b+1 \\
c+2
\end{array} \mid z\right)
\end{aligned}
$$

and then applying [30, p. 71, eqn. (1)], we obtain 


$$
\begin{aligned}
& \frac{b(c+n)(b-c) z^{2}}{c(c+1)}{ }_{2} F_{1}\left(\begin{array}{c}
-n+1, b+1 \\
c+2
\end{array} \mid z\right) \\
& =(c z-c-b z){ }_{2} F_{1}\left(\begin{array}{c}
-n, b \\
c
\end{array}\right.
\end{aligned}
$$

Replacing $b=\lambda+i x, c=2 \lambda$ and $z=2$ in (10), we obtain

$$
\frac{\left(\lambda^{2}+x^{2}\right)(2 \lambda+n)}{\lambda(2 \lambda+1)} P_{n-1}^{\lambda+1}(x)=i x P_{n}^{\lambda}(x)+\lambda P_{n-1}^{\lambda}(x), \quad n=0,1,2, \cdots
$$

Using equation (11) and Theorem 2.2, we derive the following important theorem, which will be used to prove a special case of a conjecture mentioned in [28].

Theorem 3.2. Let $\lambda>0, t \in(0,1)$ and

$0<x_{[n / 2]}<x_{[n / 2]-1}<\cdots<x_{2}<x_{1}$ be the positive zeros of $P_{n}^{\lambda}(x)$;

$0<y_{[(n-1) / 2]}<y_{[(n-1) / 2]-1}<\cdots<y_{2}<y_{1}$ be the positive zeros of $P_{n-1}^{\lambda}(x)$;

$0<t_{[(n-1) / 2]}<t_{[(n-1) / 2]-1}<\cdots<t_{2}<t_{1}$ be the positive zeros of $P_{n-1}^{\lambda+t}(x)$;

$0<z_{[(n-1) / 2]}<z_{[(n-1) / 2]-1}<\cdots<z_{2}<z_{1}$ be the positive zeros of $P_{n-1}^{\lambda+1}(x)$.

If $n$ is even, then

$$
0<x_{\left[\frac{n}{2}\right]}<y_{\left[\frac{n-1}{2}\right]}<t_{\left[\frac{n-1}{2}\right]}<z_{\left[\frac{n-1}{2}\right]}<x_{\left[\frac{n}{2}\right]-1}<\cdots<z_{2}<x_{2}<y_{1}<t_{1}<z_{1}<x_{1} ;
$$

and if $n$ is odd, then

$$
0<y_{\left[\frac{n-1}{2}\right]}<t_{\left[\frac{n-1}{2}\right]}<z_{\left[\frac{n-1}{2}\right]}<x_{\left[\frac{n}{2}\right]}<y_{\left[\frac{n-1}{2}\right]-1}<\cdots<z_{2}<x_{2}<y_{1}<t_{1}<z_{1}<x_{1}
$$

Proof. The gamma function may be defined by an infinite product (cf. [33, (6.1.3)])

$$
\Gamma(z)=\frac{\prod_{r=1}^{\infty} e^{z / r}}{z e^{\gamma z} \prod_{r=1}^{\infty}(1+z / r)}
$$

where $\gamma$ is the Euler-Mascheroni constant. Since $\Gamma(\bar{z})=\overline{\Gamma(z)}$ (cf. $[33,(6.1 .23)]$ ), it follows from (14) that

$$
|\Gamma(\lambda+i x)|^{2}=\Gamma(\lambda-i x) \Gamma(\lambda+i x)=\frac{1}{\left(\lambda^{2}+x^{2}\right) e^{2 \gamma \lambda}} \prod_{r=1}^{\infty} \frac{e^{\frac{2 \lambda}{r}}}{\left(1+\frac{\lambda}{r}\right)^{2}+\left(\frac{x}{r}\right)^{2}},
$$

which implies that

$$
\frac{|\Gamma(\lambda+1+i x)|^{2}}{|\Gamma(\lambda+t+i x)|^{2}}=e^{2 \gamma(t-1)} \frac{(\lambda+t)^{2}+x^{2}}{(\lambda+1)^{2}+x^{2}} \prod_{r=1}^{\infty} \frac{(r+\lambda+t)^{2}+x^{2}}{(r+\lambda+1)^{2}+x^{2}} \prod_{r=1}^{\infty} e^{\frac{2(1-t)}{r}} .
$$

From (15), by using the product rule, it can be seen that the ratio $w(x, \lambda+1) / w(x, \lambda+t)$, $t \in(0,1)$ is an increasing function of $x$ for $x \in(0, \infty)$, since the function $\frac{a^{2}+x^{2}}{b^{2}+x^{2}}$ is positive and increasing for $x \in(0, \infty)$ when $0<a<b$. Similarly we can show that 
$w(x, \lambda+t) / w(x, \lambda)$ is increasing for $x \in(0, \infty)$. Theorem 2.2 then implies that $y_{\nu}<t_{\nu}$ and $t_{\nu}<z_{\nu}$ for each $\nu=1,2,3, \cdots,[(n-1) / 2]$, that is

$$
y_{\nu}<t_{\nu}<z_{\nu}, \nu=1,2,3, \cdots,[(n-1) / 2] .
$$

On the other hand, it is well-known from the classic orthogonal polynomial theory that the zeros of $P_{n}^{\lambda}(x)$ and $P_{n-1}^{\lambda}$ are interlacing, that is, when $n$ is even,

$$
0<x_{[n / 2]}<y_{[n-1 / 2]}<x_{[n / 2]-1}<\cdots<x_{2}<y_{1}<x_{1} .
$$

Next, we prove that the zeros of $P_{n}^{\lambda}(x)$ interlace with those of $P_{n-1}^{\lambda+1}$. Evaluating (11) at $x=x_{k}$ and $x=x_{k+1}, k=1,2, \cdots,[(n-1) / 2]$, we obtain

$$
\left(\lambda^{2}+x_{k}^{2}\right)\left(\lambda^{2}+x_{k+1}^{2}\right)\left(\frac{2 \lambda+n}{\lambda(2 \lambda+1)}\right)^{2} P_{n-1}^{\lambda+1}\left(x_{k}\right) P_{n-1}^{\lambda+1}\left(x_{k+1}\right)=\lambda^{2} P_{n-1}^{\lambda}\left(x_{k}\right) P_{n-1}^{\lambda}\left(x_{k+1}\right)<0
$$

for each $k=1,2, \cdots,[n-1] / 2$ since the zeros of $P_{n}^{\lambda}(x)$ and $P_{n-1}^{\lambda}(x)$ interlace. So there is at least one zero of $P_{n}^{\lambda}(x)$ lying in the interval $\left(x_{k}, x_{k+1}\right)$ for each $k=1,2, \cdots,[n-1] / 2$ which implies that

$$
x_{[n / 2]}<z_{[(n-1) / 2]}<x_{[n / 2]-1}<z_{[(n-1) / 2]-1}<\cdots<z_{2}<x_{2}<z_{1}<x_{1},
$$

Now (18), (16) and (17) yield (12). The proof of (13) follows along the same lines.

Considering that when the weight function is even, the zeros of $p_{n}(x)$ are symmetric about the origin with a zero at the origin when $n$ is odd, we have the following corollary.

Corollary 3.3. With the same symbols as Theorem 3.2, we have for $n$ odd that

$$
x_{n}<y_{n-1}<t_{n-1}<z_{n-1}<x_{n-1}<\cdots<z_{2}<x_{2}<y_{1}<t_{1}<z_{1}<x_{1}
$$

while for $n$ even

$$
x_{n}<y_{n-1}<t_{n-1}<z_{n-1}<x_{n-1}<\cdots<z_{\left[\frac{n-1}{2}\right]+2}<x_{\left[\frac{n}{2}\right]+1}<0
$$

and

$$
0<x_{\left[\frac{n}{2}\right]}<y_{\left[\frac{n-1}{2}\right]}<t_{\left[\frac{n-1}{2}\right]}<z_{\left[\frac{n-1}{2}\right]}<x_{\left[\frac{n}{2}\right]-1}<\cdots<z_{1}<x_{1}
$$

with

$$
y_{\left[\frac{n-1}{2}+1\right]}=t_{\left[\frac{n-1}{2}+1\right]}=z_{\left[\frac{n-1}{2}+1\right]}=0 .
$$

In [28], the authors guess that the zeros of $P_{n}^{\lambda}(x ; \phi)$ and $P_{n-1}^{\lambda+t}(x ; \phi), t \in(0,1)$ interlace. Their conjecture is confirmed by Corollary 3.3 when $\phi=\frac{\pi}{2}$, except for the multiple zeros at the origin when $n$ is even due to the symmetry, but the general case where $0<\phi<\pi$ remains an open problem. The monotonicity of the zeros of general MeixnerPollaczek polynomials with respect to $\phi$ was proved in a recent paper by Dimitrov and Sri Ranga (cf. [34]). 


\subsection{Al-Salam-Chihara polynomials}

The Al-Salam-Chihara polynomials (cf. [35, (14.8.1)]) which we will denote by $Q_{n}(x ; a, b)$ are orthogonal on $(-1,1)$ with respect to the weight function

$$
w(x)=\frac{h(x, 1) h(x,-1) h\left(x, q^{1 / 2}\right) h\left(x,-q^{1 / 2}\right)}{h(x, a) h(x, b)}
$$

when $a, b \in \mathbb{R}$ or $a=\bar{b}$ and $\max \{|a|,|b|\}<1$ where $0<q<1$ and

$$
h(x, \alpha)=\prod_{k=0}^{\infty}\left(1-2 x \alpha q^{k}+\alpha^{2} q^{2 k}\right) .
$$

When $a=-b$,

$$
w(x, a)=\frac{h(x, 1) h(x,-1) h\left(x, q^{1 / 2}\right) h\left(x,-q^{1 / 2}\right)}{h(x, a) h(x,-a)}
$$

is an even function and we can apply Theorem 2.1. We will consider $0<a<1$ without loss of generality due to the symmetry of $w(x, a)$ with respect to $a$.

Theorem 3.4. Let $0<q<1,0<a<1,0<t<1-a$ and denote by $x_{\nu}$ and $X_{\nu}, \nu=$ $1,2, \ldots,[n / 2]$ the positive zeros in decreasing order of $Q_{n}(x ; a,-a)$ and $Q_{n}(x ; a+t,-a-t)$ respectively. Then

$$
x_{\nu}<X_{\nu} \quad \text { for } \quad \nu=1,2, \ldots,[n / 2] \text {. }
$$

Proof. The ratio of the weight functions of $Q_{n}(x ; a,-a)$ and $Q_{n}(x ; a+t,-a-t)$ is

$$
\begin{aligned}
\frac{w(x, a+t)}{w(x, a)} & =\prod_{k=0}^{\infty} \frac{\left(1+a^{2} q^{2 k}\right)^{2}-4 a^{2} q^{2 k} x^{2}}{\left(1+(a+t)^{2} q^{2 k}\right)^{2}-4(a+t)^{2} q^{2 k} x^{2}} \\
& =\left(\frac{a}{a+t}\right)^{2} \prod_{k=0}^{\infty} \frac{x^{2}-\left(\frac{1+a^{2} q^{2 k}}{2 a q^{k}}\right)^{2}}{x^{2}-\left(\frac{1+(a+t)^{2} q^{2 k}}{2(a+t) q^{k}}\right)^{2}} \\
& =\left(\frac{a}{a+t}\right)^{2} \prod_{k=0}^{\infty} \frac{x^{2}-c_{k}^{2}}{x^{2}-d_{k}^{2}}
\end{aligned}
$$

Assume that $0<q<1,0<a<1$ and $0<t<1-a$. Then, for each $k=0,1,2, \ldots$, we have that $0<(a+t) q^{k}<1$ which yields

$$
0<\left(1-(a+t) q^{k}\right)^{2}<\left(1-a q^{k}\right)^{2}<1, k=0,1,2, \ldots
$$

and, since we also know that

$$
0<\frac{1}{2(a+t) q^{k}}<\frac{1}{2 a q^{k}}, k=0,1,2, \ldots,
$$

we deduce from (19) and (20) that $d_{k}<c_{k}$ for each $k=0,1,2, \ldots$. This implies that for each $k=0,1,2, \ldots$, the function $\frac{x^{2}-c_{k}^{2}}{x^{2}-d_{k}^{2}}$ is positive and increasing for $x \in\left(-d_{k}, d_{k}\right)$. Since 
$d_{k}>1$, the ratio $\frac{w(x, a+t)}{w(x, a)}$ will be positive and increasing for $x \in(0,1)$ and the result immediately follows from Theorem 2.2.

Remark 3.5.(i) The limiting relation as $q \rightarrow 1$ for Al-Salam-Chihara polynomials that yields Meixner-Pollaczek polynomials (cf. [35, 14.8.19]) cannot be used in conjunction with Theorem 3.4 to obtain any monotonicity or interlacing results for zeros of MeixnerPollaczek polynomials since the condition $a=-b$ in Theorem 3.4 is not satisfied by the substitutions $a=q^{\lambda} e^{-i \phi}$ and $b=q^{\lambda} e^{i \phi}$, even for the special case where $\phi=\frac{\pi}{2}$.

(ii) Theorem 2.2 can also be applied, if not in general, at least to special cases of other q-orthogonal polynomials. Examples include Askey-Wilson, $q$-Meixner-Pollaczek, Continuous $q$-Jacobi, Continuous $q$-Ultraspherical and Continuous $q$-Legendre polynomials.

\section{Concluding remark}

Although Theorem 2.1 allows application to polynomials that are orthogonal with respect to a discrete weight, the well-known discrete orthogonal polynomials in the Askey and q-Askey scheme (cf. [35]) are not orthogonal on intervals that are symmetric about the origin, while other examples of discrete orthogonal polynomials such as Gram polynomials [36], Lommel polynomials [5, section 6.5] and Tricomi-Carlitz orthogonal polynomials [37] also do not satisfy the specific conditions of Theorem 2.1.

\section{Acknowledgements}

The authors would like to thank the anonymous referee for helpful comments. Research of K. Jordaan is supported by the National Research Foundation of South Africa. Research of $\mathrm{H}$ Wang is supported by the Scientific Research Project of the University of South China under grant no. 2011XQD36, the Natural Science Foundation of Hunan Province under grant no. 14JJ6020, the Construct Program in University of South China and the Hengyang Planning Project of Science and Technology under grant no. 2013KJ20. Research by J. Zhou is supported by the National Natural Science Foundation of the People's Republic of China under grant no. 11201070.

\section{References}

[1] A. Markoff, Sur les racines de certaines équations (Second note), Math. Ann., 27 (1886), $177-182$.

[2] M.E.H. Ismail, The variation of zeros of certain orthogonal polynomials, Advances in Appl. Math., 8 (1987), 111-118.

[3] M.E.H. Ismail. Monotonicity of zeros of orthogonal polynomials, in $q$-Series and Partitions, (D. Stanton, Ed), p 177-190, Springer-Verlag, New York, 1989.

[4] M.E.H Ismail, M.E. Muldoon, A discrete approach to monotonicity of zeros of orthogonal polynomials, Trans. Amer. Math. Soc., 323 (1991), 65-78.

[5] M.E.H. Ismail, Classical and Quantum Orthogonal Polynomials in One Variable, Vol. 98 of Encyclopedia of Mathematics and its Applications, Cambridge University Press, Cambridge, 2005.

[6] W. Erb and F. Toókos, Applications of the monotonicity of extremal zeros of orthogonal polynomials in interlacing and optimization problems, Appl. Math. Comput. 217, 4771-4780 (2011). 
[7] D.K. Dimitrov, On a conjecture concerning monotonicity of ultraspherical polynomials, $J$. Approx. Theory, 116 (2002), 88-97.

[8] D.K Dimitrov, F. R. Rafaeli, Monotonicity of zeros of Jacobi polynomials, J. Approx. Theory, 149 (1) (2007), 15-29.

[9] D.K Dimitrov, F. R. Rafaeli, Monotonicity of zeros of Laguerre polynomials, J. Comput. Appl. Math., 233 (3) (2009), 699-702.

[10] D.K. Dimitrov, R.O. Rodrigues, On the behaviour of zeros of Jacobi polynomials, J. Approx. Theory, 116 (2) (2002), 224-239.

[11] A. Elbert, P.D. Siafarikas, Monotonicity properties of the zeros of ultraspherical polynomials, J. Approx. Theory, 97 (1999) 31-39.

[12] M.E.H. Ismail, J Letessier, Monotonicity of zeros of ultraspherical polynomials, in Orthogonal polynomials and their applications, (M. Alfaro, J.S. Dehesa, F.J.Marcellan, J.L. Rubio de Frabcia, J. Vinuesa, Eds.), Lecture Notes in Mathematics, vol. 1329, p. 329-330, Springer, Berlin, 1988.

[13] A. Laforgia, A monotonic property for the zeros of ultraspherical polynomials, Proc. Amer. Math. Soc., 83 (1981), 757-758.

[14] G. Szegö. Orthogonal Polynomials, fourth edition. American Mathematical Society, Colloquium Publications volume 23, Providence, Rhode Island, 2003.

[15] A. Elbert, M.E. Muldoon, On the derivative with respect to a parameter of a zero of a Sturm-Liouville function, SIAM J. Math. Anal., 25 (1994), 354-364.

[16] M.E. Muldoon, Properties of zeros of orthogonal polynomials and related functions, J. Comp. Appl. Math., 48 (1993), 167-186.

[17] D.K. Dimitrov, M.V. Mello, F.R. Rafaeli, Monotonicity of zeros of Jacobi-Sobolev type orthogonal polynomials, Appl. Numer. Math., 60 (2010), 263-276.

[18] D.K. Dimitrov, M.V. Mello, F.R. Rafaeli, Monotonicity of zeros of Laguerre-Sobolev-type orthogonal polynomials, J. Math. Anal. Appl., 368 (2010), 80-89.

[19] K. Driver, K. Jordaan, Separation theorems for the zeros of certain hypergeometric polynomials, J. Comput, Appl. Math., 199 (1) (2007), 48-55.

[20] K. Driver, K. Jordaan, N. Mbuyi, Interlacing of the zeros of Jacobi polynomials with different parameters, Numer Algor., 49 (2008), 143-152.

[21] F. Marcellán, F.R. Rafaeli, Monotonicity and Asymptotics of zeros of Laguerre-Sobolev-type orthogonal polynomials of higher order derivatives, Proc. Amer. Math. Soc., 139(11) (2011), 3929-3936.

[22] G. Freud, Orthogonal Polynomials, Pergamon, Oxford, 1971. [English translation]

[23] D.K. Dimitrov, A. Sri Ranga, Monotonicity of the zeros of orthogonal Laurent polynomials, Methods Appl. Anal., 9 (2002), 1-12.

[24] M.E.H. Ismail, J Letessier, D.R. Masson, G. Valent, Birth and Death processes and orthogonal polynomials, in Orthogonal polynomials: Theory and Practice (P. Nevai Ed.), NATO ASI Series, Kluwer Academic Publishers, Dordrecht, Boston and London, 1990, p. 229-255.

[25] H. Kiesel, J. Wimp, A note on Koornwinder's polynomials with weight function $(1-x)^{\alpha}(1+$ $x)^{\beta}+M \delta(x+1)+N \delta(x-1)$, Numer. Algor., 11 (1996), 229-241.

[26] T.H. Koornwinder, Orthogonal polynomials with weight function $(1-x)^{\alpha}(1+x)^{\beta}+M \delta(x+$ 1) $+N \delta(x-1)$, Canad. Math. Bull., 27(2) (1994), 205-214.

[27] T.J. Stieltjes, Sur les racines de l'equation $X_{n}=0$. Acta Math., 9 (1886), 385-400. Oeuvres Complétes. Vol. 2, pp.73-88.

[28] K. Jordaan, F. Toókos, Interlacing theorems for the zeros of some orthogonal polynomials from different sequences, Appl. Numer. Math., 59 (2009), 2015-2022.

[29] D. Dominici, K. Driver, K. Jordaan, Polynomial solutions of differential-difference equations, J. Approx. Theory, 163 (2011), 41-48.

[30] E D Rainville. Special Functions. (The Macmillan Company, New York, 1960)

[31] K. Driver, A. Jooste, K. Jordaan, Stieltjes interlacing of zeros of Jacobi polynomials from different sequences, Electron. Trans. Numer. Anal., 38 (2011) 317-326.

[32] R. Vidũnas and T. Koornwinder, Webpage of the NWO project, Algorithmic methods for special functions by computer algebra, http://www.science.uva.nl/ thk/specfun/compalg.html, 2000.

[33] M. Abramowitz, I. Stegun, Handbook of Mathematical Functions, (Dover, New York, 1965). 


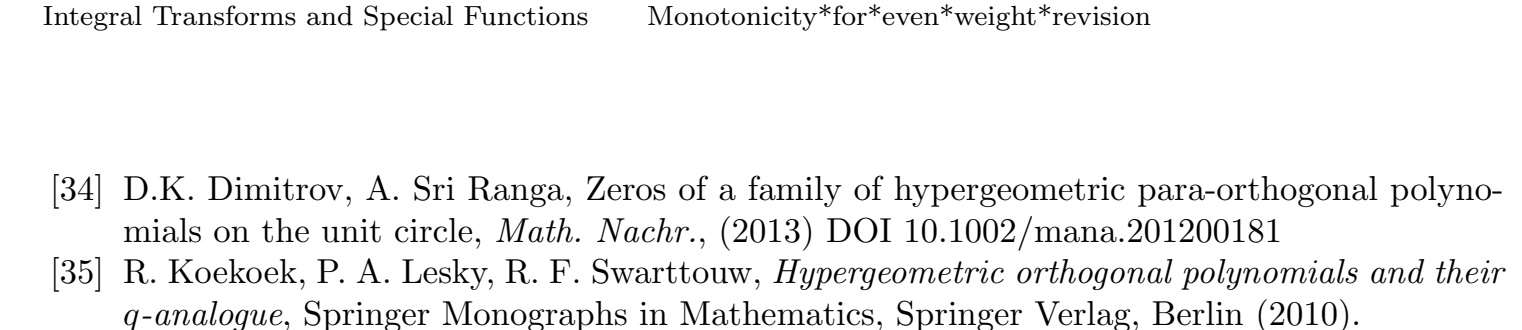

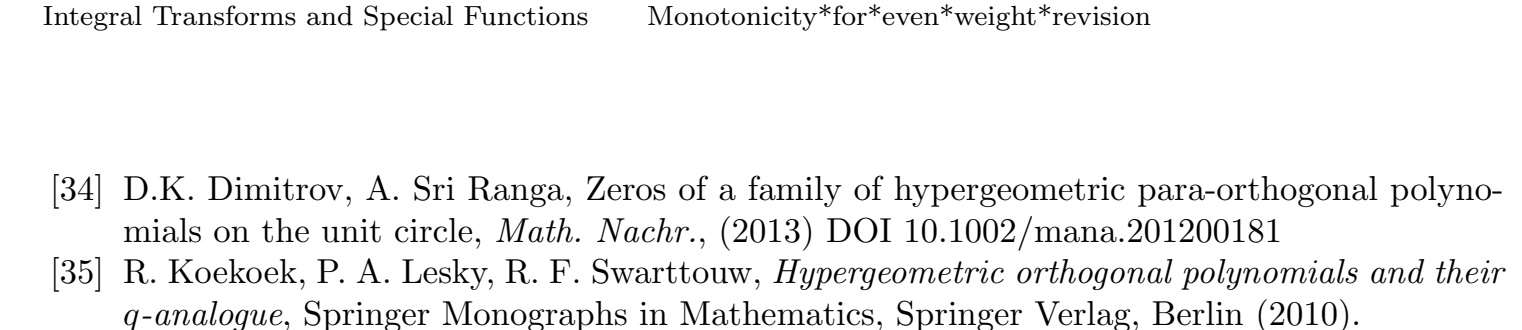

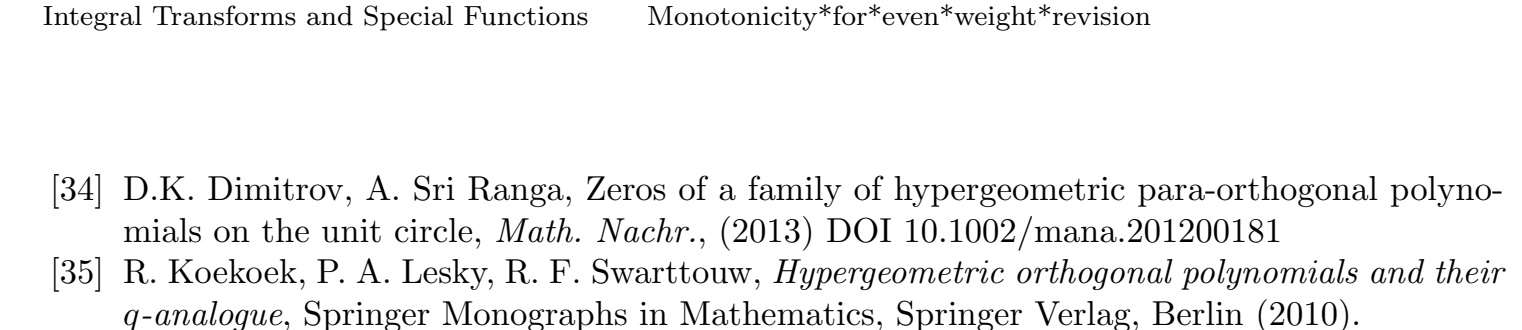

Integral Transforms and Special Functions Monotonicity*for*even*weight*revision
$\begin{aligned} & \text { [34] D.K. Dimitrov, A. Sri Ranga, Zeros of a family of hypergeometric para-orthogonal polyno- } \\ & \text { mials on the unit circle, Math. Nachr., (2013) DOI 10.1002/mana.201200181 } \\ & \text { [35] R. Koekoek, P. A. Lesky, R. F. Swarttouw, Hypergeometric orthogonal polynomials and their } \\ & \text { q-analogue, Springer Monographs in Mathematics, Springer Verlag, Berlin (2010). }\end{aligned}$

[36] F. B. Hildebrand, Introduction to Numerical Analysis, Dover, New York, 1987.

[37] L. Carlitz, On some polynomials of Tricomi, Boll. Un. Mat. Ital., 13(1958), 58-64, 37] L. Carlitz, On some polynomials of Tricomi, Boll. Un. Mat. Ital., 13(1958), 58-64,
MR0103303.(21:2078).

coras 